\title{
Review of $e^{+} e^{-} \rightarrow \pi^{+} \pi^{-} \eta$
}

\author{
Olga Shekhovtsova ${ }^{1,2, \star}$ \\ ${ }^{1}$ NSC KIPT Akhiezer Institute for theoretical Physics, 61108 Kharkov, Ukraine \\ ${ }^{2}$ Institute of Nuclear Physics PAN, 31-234 Cracow, Poland
}

\begin{abstract}
In this talk we review the current experimental status of electron-positron annihilation into $\pi^{+} \pi^{-} \eta$ as well as the theoretical models to describe the data. Application of the CVC hypothesis to get the $\tau^{-}$decay to $\pi^{-} \pi^{0} \eta$ branching fraction is discussed and its values from the different experiments is listed.
\end{abstract}

\section{Introduction}

The data on the $e+e^{-}$annihilation in the energy region below $2 .-2.5 \mathrm{GeV}$ is the main source of information about properties of the light vector meson and is of great interest for a better understanding of the light quark interactions. For the process

$$
e^{+}+e^{-} \rightarrow \pi^{+}+\pi^{-}+\eta
$$

the main mechanism of production proceeds through one intermediate state $\eta \rho(770){ }^{1}$ which makes the precise measurements of the cross section of the reaction (1) of great importance for the determination of the $\rho(1400)$ and $\rho(1700)$ parameters. Also data on $e^{+} e^{-} \rightarrow \pi^{+} \pi^{-} \eta$ can be used to test the conservation of the vector current (CVC) hypothesis.

The reaction (1) was first observed at the end of 70's in the DM1 experiment [1] and since that time it was studied by Orsay [2], Novosibirsk [3-6] groups and by the BaBar collaboration [7, 8]. From a theoretical point of view the $\eta \pi \pi$ production in the $e^{+} e^{-}$-annihilation and $\tau$-lepton decay was discussed in [4, 9-11] using different approaches.

The paper is organized as follows. In Sec. 2 the theoretical approaches to describe hadronic currents $\gamma^{*} \rightarrow \pi^{+} \pi^{-} \eta$ are described. The experimental data are reviewed in Sec. 3. Sec. 4 is devoted to the CVC study and comparison with the spectral function of the $\tau^{-} \rightarrow \pi^{-} \pi^{0} \eta v_{\tau}$ decay. The rare decay $\phi \rightarrow \pi^{+} \pi^{-} \eta$ is the subject of Sec 5 .

\section{Theoretical models}

The description of hadronic interactions in the energy region populated by resonances is problematic. In fact, perturbative QCD does not work in the region below $2 \mathrm{GeV}$. At very low energies $(E<<$ $M_{\rho}$ ), Chiral Perturbation Theory (ChPT), based on the chiral symmetry of massless QCD, provides

^e-mail: oshekhov@ifj.edu.pl

${ }^{1}$ A production via $e^{+} e^{-} \rightarrow a 2(1300) \pi$ can contribute to the cross section, however, this reaction has not been observed. 
successful description of hadronic interactions. At higher energy there is no a similar framework and we have to rely on some phenomenological models. Three theoretical models have been applied to study the process (1) and they are shortly described below.

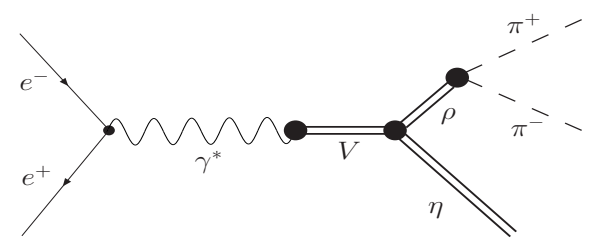

Figure 1. The diagram of the $e^{+} e^{-} \rightarrow \eta \pi^{+} \pi^{-}$process in the framework of the VMD model.

The Vector Meson Dominance (VMD) model with three intermediate $\rho$ meson states is a generally accepted scheme in the contemporary $\eta \pi \pi$ data analysis. It is characterized by the Feynman diagram shown in Fig. 1, where the intermediate resonance $V$ represents the mesons $\rho(770), \rho(1450), \rho(1700)$. The corresponding cross section can be written as [5]

$$
\sigma(s)=\frac{4 \alpha^{2}}{3} \frac{1}{s \sqrt{s}} \int_{4 m_{\pi}^{2}}^{\left(\sqrt{s}-m_{\eta}\right)^{2}} \frac{\sqrt{q^{2}} \Gamma_{\rho}\left(q^{2}\right) P_{\eta}^{3}\left(s, q^{2}\right)}{\left(q^{2}-m_{\rho}^{2}\right)^{2}+\left(\sqrt{q^{2}} \Gamma_{\rho}\left(q^{2}\right)\right)^{2}}|F(s)|^{2} d q^{2},
$$

where $q$ is the 4-momentum of the $\pi^{+} \pi^{-}$system and $P_{\eta}^{2}\left(s, q^{2}\right)=\left[\left(s-m_{\eta}^{2}-q^{2}\right)^{2}-4 m_{\eta}^{2} q^{2}\right] / 4 s$.

The transition form factor $F(s)$ describes the vertex $\gamma^{*} \rightarrow \eta \rho$ and takes the form:

$$
F(s)=\sum_{V} \frac{g_{V} e^{i \phi_{V}} m_{V}^{2}}{s-m_{V}^{2}+i \sqrt{s} \Gamma_{V}(s)},
$$

where $g_{V} e^{i \phi_{V}}=g_{V \rho \eta} / g_{V \gamma}, g_{V \rho \eta}$ and $g_{V \gamma}$ are the coupling constants for the transitions $V \rightarrow \rho \eta$ and $V \rightarrow \gamma^{*}$, respectively. Here the energy dependence of the $\rho$ meson widths is driven by two-pion loops $\Gamma_{V}\left(q^{2}\right)=\Gamma_{V} \frac{m_{V}^{2}}{q^{2}}\left[p_{\pi}\left(q^{2}\right) / p_{\pi}\left(m_{V}^{2}\right)\right]^{3}, p_{\pi}\left(q^{2}\right)=\sqrt{q^{2}-4 m_{\pi}^{2}}$.

Since the coupling constants are not expected to have sizable imaginary parts, the fit to the experimental data is done assuming the phases $\phi_{\rho(1450)}$ and $\phi_{\rho(1700)}$ equal to zero or $\pi$ (if the reference $\rho(770)$ phase is put equal to zero). The SND Collaboration analysis [5] favours $\phi_{\rho(1450)}=\phi_{\rho(1700)}=\pi$. Notice that the fit with $\rho(1700)$ is slightly better than without it; however, the value obtained for $g_{\rho(1700)}$ deviates from zero by only $2 \sigma$.

The description of the process (1) within the framework of the Resonance Chiral Theory (RChT) is presented in $[9,10]$. RChT is based on the ChPT approach with the explicit inclusion of the vector and axial-vector resonances. Its main features can be summarized in two points: 1) the RChT Lagrangian contains the ChPT one and hence the RChT amplitudes satisfy chiral symmetry and match the chiral amplitudes at low energies; 2) the high-energy amplitude of the hadronic form factors provided by RChT is suppressed, as enforced by QCD.

The $\eta \pi \pi$ production in the RChT framework is described by the diagrams in Fig. 3. Leaving only the diagram 4d), we obtain the VMD approach. In Ref. [9], in addition to the photon propagation, only the lightest $\rho(770)$ resonance was considered. Comparison with experimental data [1-4, 7] demonstrates fair agreement in the energy region from the $\eta \rho$ threshold to $1.4 \mathrm{GeV}$ (Fig. 4, left 


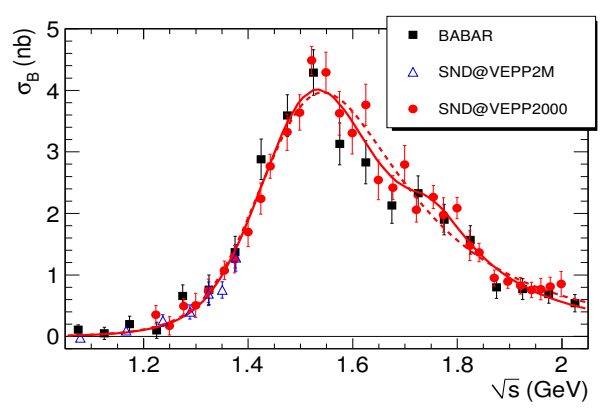

Figure 2. The cross section $e^{+} e^{-} \rightarrow \pi^{+} \pi^{-} \eta$ measured in the SND experiment: the solid line is the VMD parametrization including $\rho(1700)$, the dashed line without it. The figure is taken from [5].
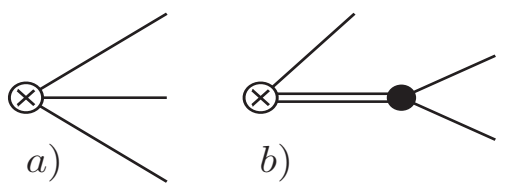

b)

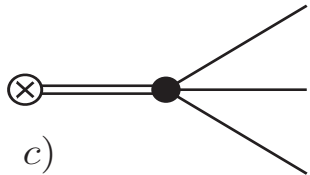

c)

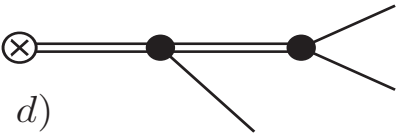

Figure 3. Hadronization of the vector current in $e^{+} e^{-} \rightarrow \pi^{+} \pi^{-} \eta$ within the RChT approach. Single lines represent pseudoscalar mesons $(\pi, \eta)$ and double lines are for the $\rho$ resonances. The diagram a) corresponds to the Wess-Zumino-Witten interaction Lagrangian, the diagrams b) c) and d) to the effective Lagrangian describing interactions with resonances.

panel). An extended approach to study the reaction $e^{+} e^{-} \rightarrow \pi^{+} \pi^{-} \eta\left(\pi^{0}\right)$, including the excited $\rho(1450)$ and $\rho(1700)$ mesons as well as tiny isospin symmetry breaking effects, was proposed in [10]. In order to obtain the values of the model parameter, fits based on different sets of $e^{+} e^{-} \rightarrow \pi^{+} \pi^{-} \pi^{0}$ and $e^{+} e^{-} \rightarrow \pi^{+} \pi^{-} \eta$ data were carried out. For details, see Section VII of [10]. Comparison between the theoretical prediction for the cross section of process (1) and the experimental data is presented in Fig. 4, right panel. Notice, in addition, that the fit favors an higher mass for the $\rho(1450)$ resonance $\left(M_{\rho^{\prime}}=1.55 \mathrm{GeV}\right.$ instead of its PDG value $\left.M_{\rho^{\prime}}=1.465(25) \mathrm{GeV}\right)$ and lower width $\left(\Gamma_{\rho^{\prime}}=0.238 \mathrm{GeV}\right.$ instead of its PDG value $\left.\Gamma_{\rho^{\prime}}=0.400(60) \mathrm{GeV}\right)$. Also the contribution of the $\rho(1700)$ is one half that of the $\rho(1450)$.

The reaction (1) within the extended nonlocal version of the Nambu-Jona-Lasinio (NJL) model studied in $[11,12]$ and the corresponding Feynman diagrams are shown in Fig. 5. The standard NJL model describes the physical processes via an intermediate states of a virtual photon and $\rho$ and $\omega$ mesons in its ground state. The contribution of the first radially excited meson states is included in the extended NJL model [13] and provides a reasonable good description of a wide range of physical process for energy region up to about $1.5 \mathrm{GeV}$ [12].

Hadronization mechanism to produce $\eta \pi \pi$ is presented in Fig. 6. The VMD approach could be directly obtained from the NJL model by taking into account only the diagram with the triangle loops. Precise formulae for the cross section can be found in Section III of [11]. Numerical values of the model parameters are fixed in Eq.(4) of [11] whereas for the resonance masses and widths their PDG values are chosen. The comparison with the BaBar data is presented in Fig. 7. 

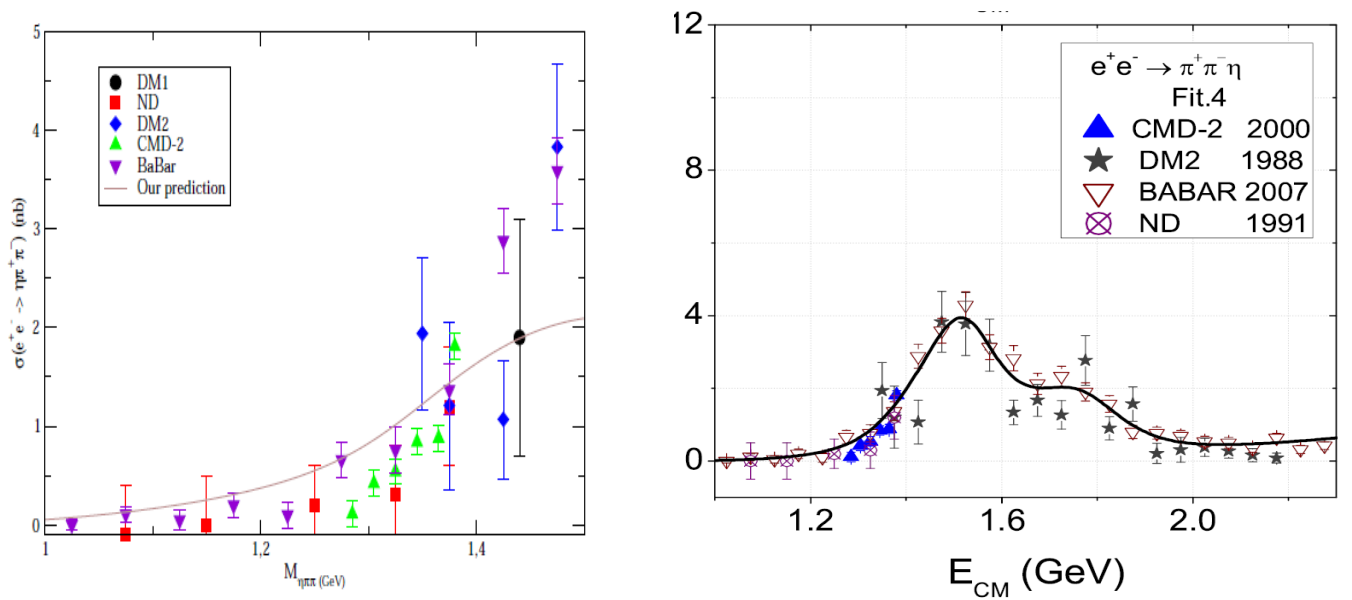

Figure 4. Comparison between the experimental data for $e^{+} e^{-} \rightarrow \pi^{+} \pi^{-} \eta$ and the theoretical prediction based on the RChT approach. The left panel corresponds to the propagation via $\gamma+\rho(770)$, the right one to the elaborated model used in Ref. [10]. The figures are taken from $[9,10]$.
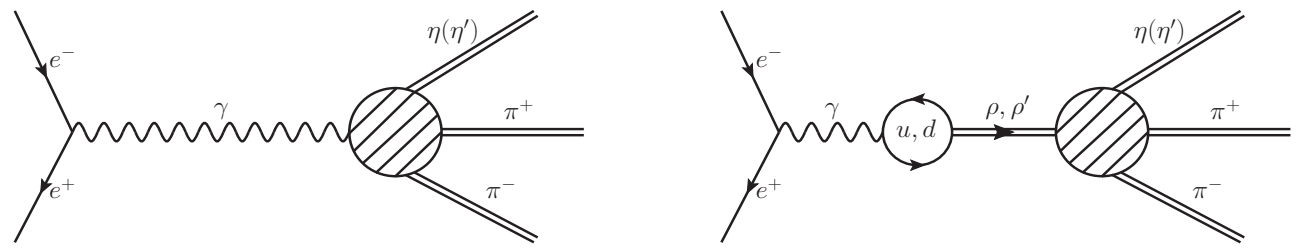

Figure 5. Electron-positron annihilation with $\eta \pi \pi$ production within the NJL approach. The left diagram corresponds to the production via virtual photons, the right one is via intermediate $\rho(770), \rho(1450)$ states. The dashed circles are the sum of two subdiagrams from Fig. 6.
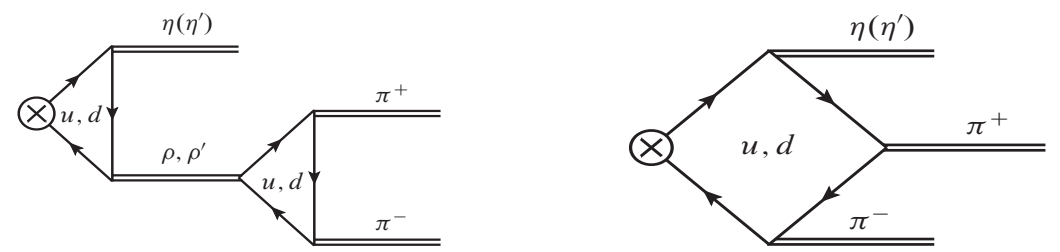

Figure 6. Vertex subdiagrams $V \eta \pi \pi$ with a quark box loop (left) and with two quark-triangle loops (right).

\section{Experimental data}

As the $\eta$ meson is a short lifetime particle it is observed decaying either in $\pi^{+} \pi^{-} \pi^{0}$ or via its $\gamma \gamma$ decay. Therefore the process (1) contributes to the reactions: $e^{+} e^{-} \rightarrow \pi^{+} \pi^{-} \pi^{0} \pi^{+} \pi^{-}$and $e^{+} e^{-} \rightarrow \pi^{+} \pi^{-} \gamma \gamma$. 


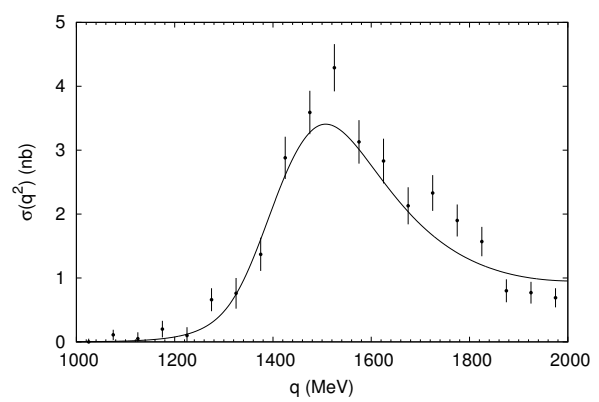

Figure 7. Comparison of the extended NJL model prediction with the BaBar experiment data [7]. The figure is taken from [11].

The reaction (1) was first observed at the end of the 70's in the DM1 experiment $[1]^{2}$ for the centerof-mass $(\mathrm{CM})$ energy region $1.4-2.8 \mathrm{GeV}$. The DM1 experiment data analysis demonstrated that the $\rho \eta$ production only via the intermediate $\rho(770)$ resonance [15], predicts a cross section of about 0.5 $\mathrm{nb}$, an order of magnitude below the experimental data. To solve the problem, the production via the intermediate $\rho^{\prime}(1600)$ was added. The ND Collaboration [3] studied the process in the CM energy region $1.05-1.40 \mathrm{GeV}$ and showed the same excess of production compared with the theoretical prediction [15] for energies above $1.35 \mathrm{GeV}$, see Fig. 8, confirming the hypothesis that the main contribution in this energy region comes from the intermediate $\rho^{\prime}(1600)$ meson.

Further comparison of the $\eta \rho$ mass spectrum in diffractive photoproduction [16] and $e^{+} e^{-}$annihilation has brought to the idea of the existence of two $\rho^{\prime}$ resonances with a mass of $1.47 \mathrm{GeV}$ and $1.70 \mathrm{GeV}$ [17]. This parametrization has been described in Sec. 2 and since the DM2 experiment it is the generally accepted pattern in the $\eta \pi \pi$ data analysis.

Later the process was studied by Orsay (DM2 Collaboration) [2] and Novosibirsk groups (SND, CMD groups) [3, 4]. The most recent measurements were performed at the VEPP-2000 collider by the SND group [5] for the CM energy range from 1.22 to $2.00 \mathrm{GeV}$ (the $\eta$ meson is recontructed via the two-photon mode) and at B-factory with the BaBar detector using the initial state radiation technique [7] in the $(1.025 ; 2.975) \mathrm{GeV} \mathrm{CM}$ energy region (the $\eta$ meson is recontructed via three-pion mode). Systematic uncertainties of about $8 \%$ at BaBar [7] and below $8.3 \%$ by the SND experiment were reported. The preliminary results from BaBar using the $\eta \rightarrow \gamma \gamma$ channel [8] and from the CMD-3 collaboration using both $\eta \rightarrow \pi^{+} \pi^{-} \pi^{0}$ and $\eta \rightarrow \gamma \gamma$ decay modes have been presented recently [6, 8].

As seen from Figs. 2, 9 all measurements are consistent. The cross section shows a steep rise starting from the $\eta \rho(770)$ threshold, peaking at about $1.5 \mathrm{GeV}$, and then decreases with increasing energy. The measured $e^{+} e^{-} \rightarrow \eta \pi^{+} \pi^{-}$cross section was fitted using the VMD model (see Section 2) with and without $\rho(1700)$ contribution. Based on the fit results one cannot draw a definite conclusion that at the current statistics the $\rho(1700)$ contribution is needed for the description of the data.

\section{Check of the CVC hypothesis}

The data on electron-positron annihilation with $\eta \pi \pi$ production can be used to estimate the branching fraction of the $\tau^{-} \rightarrow \eta \pi^{-} \pi^{0} \eta v_{\tau}$ decay [19] and test of the CVC hypothesis.

\footnotetext{
${ }^{2}$ In fact the $e^{+} e^{-}$-annihilation into five pions was observed by the M3N collaboration [14], however, the intermediate mechanisms producing the final state were not studied.
} 


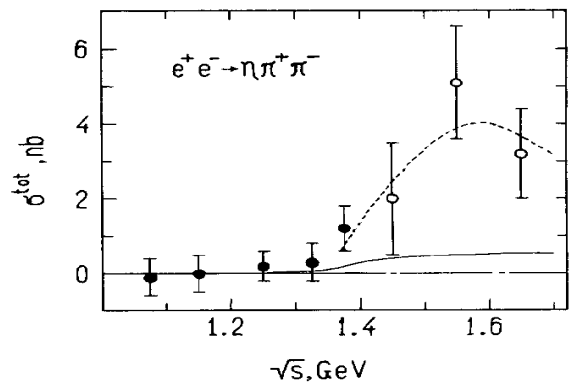

Figure 8. Energy dependence of the $e^{+} e^{-} \rightarrow \eta \pi^{+} \pi^{-}$cross section. In the VMD model, the solid line includes only the $\rho(770)$ resonance, whereas the dashed line takes into account also the $\rho(1600)$. The ND data are represented by open circles, the DM1 ones by black circles. The figure is taken from [3].
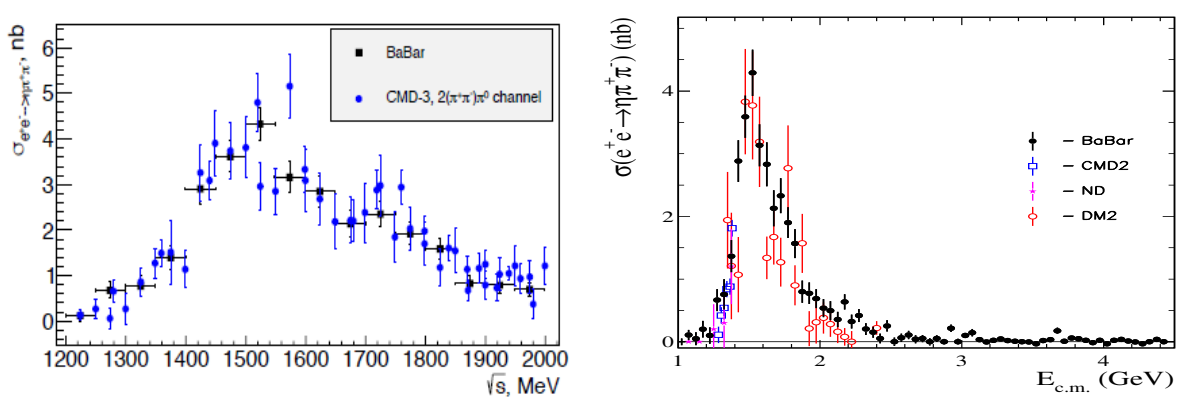

Figure 9. Comparison between experimental data for the process $e^{+} e^{-} \rightarrow \eta \pi \pi$ done by CMD-3(left panel) and BaBar Collaborations (right panel). The figures are taken from [6, 7].

The hypothesis of conserved vector current [18] and isospin symmetry is based on two assumptions: first, it assumes that the isovector part of the electromagnetic current and the vector part of the hadronic weak current are only different components of the same isospin current and, second, that the three components of this isospin current are conserved. As a consequence, hadronic currents describing vector $\tau$ decays and hadronic low energy (up to the $\tau$ lepton mass) $e^{+} e^{-}$-annihilation are related and can be obtained one from the other.

Applying CVC for the $e^{+} e^{-} \rightarrow \pi^{+} \pi^{-} \eta$ data one obtains the branching fraction of the $\tau^{-} \rightarrow \pi^{-} \pi^{0} \eta v_{\tau}$ decay:

$$
\frac{B r\left(\tau^{-} \rightarrow \pi^{-} \pi^{0} \eta v_{\tau}\right)}{B r\left(\tau^{-} \rightarrow e^{-} v_{\tau}\right)}=\frac{3\left|V_{u d}^{2}\right| S_{E W}}{2 \pi \alpha^{2}} \int_{4 m_{\pi}^{2}}^{\infty} d q^{2} \frac{q^{2}}{m_{\tau}^{2}}\left(1-\frac{q^{2}}{m_{\tau}^{2}}\right)^{2}\left(1+\frac{2 q^{2}}{m_{\tau}^{2}}\right) \sigma_{e^{+} e^{-} \rightarrow \eta \pi \pi}\left(q^{2}\right)
$$

where $S_{E W}$ is an electroweak correction and the $V_{u d}$ is the $u d$ element of the Cabibbo-KabayashiMaskawa matrix. The estimate for $\operatorname{Br}\left(\left(\tau^{-} \rightarrow \pi^{-} \pi^{0} \eta v_{\tau}\right)\right.$ is summarized in Table 1. As one can see the branching ratio derived from the $e^{+} e^{-}$CMD-2 and old data data is in agreement with the world average experimental value whereas the predicted BaBar value is slightly above it. A possible source of this difference might be an yet unknown isospin breaking corrections. 


\begin{tabular}{lll}
\hline Group & $\mathrm{Br}, \%$ & Ref. \\
\hline Old $e^{+} e^{-}$data & $0.13 \pm 0.015$ & {$[19]$} \\
BaBar 2007 & $0.165 \pm 0.015$ & {$[19]$} \\
Old $e^{+} e^{-}+$BaBar 2007 & $0.153 \pm 0.018$ & {$[19]$} \\
BaBar 2016 & $0.160 \pm 0.009$ & {$[8]$} \\
BaBar 2007+2016 & $0.162 \pm 0.008$ & {$[8]$} \\
CMD-2 & $0.156 \pm 0.004 \pm 0.01$ & {$[4]$} \\
\hline PDG & $0.139 \pm 0.007$ & {$[20]$} \\
\hline
\end{tabular}

Table 1. $\operatorname{Br}\left(\tau^{-} \rightarrow \pi^{-} \pi^{0} \eta v_{\tau}\right)$ value obtained on the base of the CVC hypothesis from $e^{+} e^{-}$data and its world average experimental value.

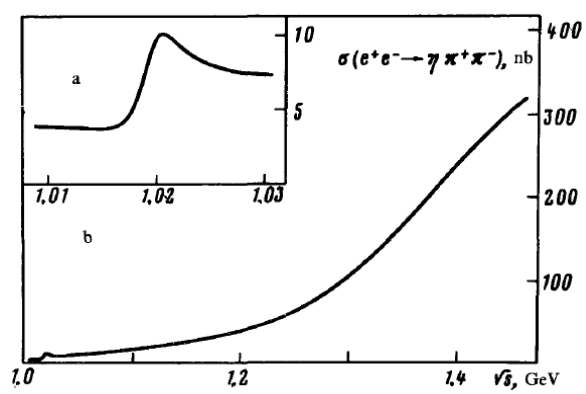

Figure 10. The VMD predicted cross section for $e^{+} e^{-} \rightarrow \eta \pi \pi$ and the influence related with production $\phi \rightarrow \eta \pi \pi$. The figure is taken from [21].

\section{Rare decay $e+e^{-} \rightarrow \phi \rightarrow \eta \pi^{+} \pi^{-}$}

Investigation of the energy dependence of the process (1) cross section for the CM energy near the $\phi$ meson mass is necessary for the search of the rare $\phi \rightarrow \eta \pi^{+} \pi^{-}$decay. This latter is both OZI suppressed and G-parity violating. Its branching ratio has been theoretically estimated within VMD: $\operatorname{Br}\left(\phi \rightarrow \eta \pi^{+} \pi^{-}\right)=0.35 \cdot 10^{-6}[21]$ and its influence on the $\eta \pi^{+} \pi^{-}$cross section is shown in Fig. 10. Experimentally the upper limit was measured by the CMD-2 Collaboration and is $\operatorname{Br}\left(\phi \rightarrow \eta \pi^{+} \pi^{-}\right)<$ $1.8 \cdot 10^{-5} 90 \%$ CL [22]. Therefore the current experimental upper limit is about 50 times above than its theoretical prediction.

\section{Conclusion}

Summarizing, during the last decade the process of $e^{+} e^{-} \rightarrow \eta \pi^{+} \pi^{-}$has been studied both by theoreticians, using the description based on the Resonance Chiral Lagrangian or the extended NambuJona-Lasinio model, and by experimental collaborations. The data sets at energies $1 .-2$. GeV have been collected in $e^{+} e^{-}$-annihilation by the CMD and SND Collaborations (Novosibirsk, VEPP-2M collider) and using the radiative return technique at the SLAC B-factory with the BaBar detector. The systematic uncertainty has been decreased substantially compared with the old experiments and 
reached the level below $8 \%$ (the preliminary analysis by CMD-3 claims even a 5\% level). The data of the three experiments are in agreement. However, a more detailed study based on the cross section approximation proposed in Ref. [19] might be applied for a better comparison between the data in whole energy region.

To fit the measured cross section, the VMD model with three isovector states $\rho(770), \rho(1450)$ and $\rho(1700)$ has been used. However, the currents statistics does not allow to conclude whether the $\rho(1700)$ is necessary to reproduce the data or new more precise measurements at energies above $1.4 \mathrm{GeV}$ would be necessary to study the region in detail. This study will require an estimate of a background related with the production via $a_{2}(1320), \rho(1400 ; 1700) \rightarrow a_{2} \pi$, but to our knowledge the corresponding process has not yet been calculated theoretically.

Also new measurements at low energies near the $\phi$ meson region are important to improve our knowledge about the rare decay $\phi \rightarrow \eta \pi^{+} \pi^{-}$(the upper experimental limit is 50 times higher than the theoretical prediction). This is planned to be done at the KLOE-2 experiment.

Assuming the isospin invariance the $e^{+} e^{-} \rightarrow \eta \pi \pi$ cross section via the CVC relation predicts the branching ratio $\tau^{-} \rightarrow \eta \pi^{-} \pi^{-} v_{\tau}$. Therefore comparison between the predicted value and its measured value would allow to test the CVC hypothesis. Currently the branching ratio derived from the CMD-2 and old $e^{+} e^{-}$data is in agreement with the average experimental value whereas the predicted value based on BaBar data is slightly above it. A possible source of this difference might be due to not yet calculated isospin-breaking corrections.

Last but not least, the $\eta \pi \pi$ contribution gives about $5 \%$ to the total hadronic cross section at energy $\sqrt{s}=1.5 \mathrm{GeV}$ and therefore gives a non-negligible contribution to the anomalous magnetic moment of muon, $(g-2)_{\mu}$.

\section{References}

[1] B. Delcourt, D. Bisello, J. C. Bizot, J. Buon, A. Cordier and F. Mane, Phys. Lett. 113B, 93 (1982) Erratum: [Phys. Lett. 115B, 503 (1982)].

[2] A. Antonelli et al. [DM2 Collaboration], Phys. Lett. B 212, 133 (1988).

[3] V. P. Druzhinin et al., Phys. Lett. B 174, 115 (1986).

[4] R. R. Akhmetshin et al. [CMD-2 Collaboration], Phys. Lett. B 489, 125 (2000).

[5] V. M. Aulchenko et al. [SND Collaboration], Phys. Rev. D 91, 052013 (2015).

[6] E. P. Solodov et al., AIP Conf. Proc. 1735, 020005 (2016).

[7] B. Aubert et al. [BaBar Collaboration], Phys. Rev. D 76, 092005 (2007)

Erratum: [Phys. Rev. D 77, 119902 (2008)].

[8] R. Waldi [BaBar Collaboration], Talk atICHEP 2016, http://indico.cern.ch/event/432527/contributions/1072438/.

[9] D. Gomez Dumm and P. Roig, Phys. Rev. D 86, 076009 (2012).

[10] L. Y. Dai, J. Portoles and O. Shekhovtsova, Phys. Rev. D 88, 056001 (2013).

[11] M. K. Volkov, A. B. Arbuzov and D. G. Kostunin, Phys. Rev. C 89, 015202 (2014).

[12] M. K. Volkov and A. B. Arbuzov, Phys. Part. Nucl. 47, 489 (2016).

[13] M. K. Volkov and V. L. Yudichev, Phys. Part. Nucl. 31,282 (2000);

M. K. Volkov, D. Ebert and M. Nagy, Int. J. Mod. Phys. A 13, 5443 (1998).

[14] G. Cosme et al., Nucl. Phys. B 152, 215 (1979).

[15] J. Layssac and F. M. Renard, Nuovo Cim. A 6, 134 (1971).

[16] M. Atkinson et al. [Omega Photon Collaboration], Nucl. Phys. B 242,269 (1984);

M. Atkinson et al. [Omega Photon Collaboration], Z. Phys. C 30, 531 (1986). 
[17] A. Donnachie and A. B. Clegg, Z. Phys. C 34, 257 (1987).

[18] H. B. Thacker and J. J. Sakurai, Phys. Lett. 36B, 103 (1971);

Y. S. Tsai, Phys. Rev. D 4, 2821 (1971).

[19] V. Cherepanov and S. Eidelman, Nucl. Phys. Proc. Suppl. 218, 231 (2011).

[20] C. Patrignani et al. [Particle Data Group], Chin. Phys. C 40, 100001 (2016).

[21] N. N. Achasov and V. A. Karnakov, JETP Lett. 39, 342 (1984).

[22] R. R. Akhmetshin et al. [CMD-2 Collaboration], Phys. Lett. B 491, 81 (2000). 